\title{
O uso da dieta DASH (Dietary Approaches to Stop Hypertension) para manejo da hipertensão arterial na atenção primária
}

The use of the DASH diet (Dietary Approaches to Stop Hypertension) to manage arterial hypertension in primary care

\section{El uso de la dieta DASH (Dietary Approaches to Stop Hypertension) para controlar la hipertensión arterial en atención primaria}

Laryssa de Lacerda Rodrigues ${ }^{1 *}$, Isadora Thamires Pacheco Celestino ${ }^{1}$, Amanda Mendes Rocha ${ }^{2}$, Beatriz Mendes Rocha ${ }^{2}$, Igor Wajnsztok Brasileiro ${ }^{1}$, Marina Mattuella Debenetti ${ }^{3}$, Nathalia Callegari Peraro², Samantha Araújo Vieira $^{2}$.

\section{RESUMO}

Objetivo: Comprovar a eficácia do uso da dieta DASH como medida complementar no manejo da hipertensão arterial em pacientes da atenção primária, não excluindo a necessidade do tratamento medicamentoso. Revisão bibliográfica: Quando comparada a outras dietas, a dieta DASH apresentou resultados expressivos na redução da pressão arterial sistólica e diastólica. Concomitantemente, foram observados redução do risco cardiovascular em decorrência do controle pressórico e da melhora de parâmetros em outros fatores de risco como sobrepeso/obesidade e perfil lipídico. Paralelamente, os estudos não concordantes com esse desfecho possuíam viesses importantes como a grande semelhança entre as dietas do grupo estudo e do grupo controle. Considerações finais: A dieta DASH se mostrou uma importante estratégia de manejo da hipertensão arterial sistêmica na saúde primária, prevenindo os fatores de risco e atuando em conjunto com o tratamento habitual dessa morbidade. Em concordância com esse aspecto, é recomendada por diversas diretrizes nacionais e internacionais, sendo a sua implementação potencialmente benéfica tanto na prevenção quanto no controle da hipertensão arterial.

Palavras-chave: Hipertensão, Abordagens dietéticas para conter a hipertensão, Saúde pública, Dieta mediterrânea.

\begin{abstract}
Objective: To prove the effectiveness of using the DASH diet as a complementary measure in the management of arterial hypertension in primary care patients, not excluding the need for drug treatment. Bibliographic review: When compared to other diets, the DASH diet showed significant results in reducing systolic and diastolic blood pressure. Concomitantly, a reduction in cardiovascular risk was observed due to blood pressure control and improved parameters in other risk factors such as overweight / obesity and lipid profile. In parallel, studies that did not agree with this outcome had important biases, such as the great similarity between the diets of the study group and the control group. Final considerations: The DASH diet proved to be an important strategy for the management of systemic arterial hypertension in primary health, preventing risk factors and acting in conjunction with the usual treatment of this morbidity. In accordance with this aspect, it is recommended by several national and international guidelines, and its implementation is potentially beneficial both in the prevention and control of arterial hypertension.
\end{abstract}

Keywords: Hypertension, Dietary approaches to stop hypertension, Public health, Diet mediterranean.

\footnotetext{
${ }_{1}$ Pontifícia Universidade Católica de Minas Gerais (PUC-MG), Betim - MG.

*E-mail: Laryssadelacerdarod@gmail.com

2 Universidade Alzira Velano (UNIFENAS), Alfenas - MG.

${ }^{3}$ Universidade de Caxias do Sul (UCS), Caxias do Sul - RS.
} 


\section{RESUMEN}

Objetivo: demostrar la efectividad del uso de la dieta DASH como una medida complementaria en el tratamiento de la hipertensión arterial en pacientes de atención primaria, sin excluir la necesidad de tratamiento farmacológico. Revisión bibliográfica: en comparación con otras dietas, la dieta DASH mostró resultados significativos en la reducción de la presión arterial sistólica y diastólica. Al mismo tiempo, se observó una reducción en el riesgo cardiovascular debido al control de la presión arterial y la mejora de los parámetros en otros factores de riesgo, como el sobrepeso / obesidad y el perfil lipídico. Al mismo tiempo, los estudios que no estaban de acuerdo con este resultado tenían sesgos importantes, como la gran similitud entre las dietas del grupo de estudio y el grupo de control. Consideraciones finales: La dieta DASH demostró ser una estrategia importante para el manejo de la hipertensión arterial sistémica en la salud primaria, previniendo los factores de riesgo y actuando en conjunto con el tratamiento habitual de esta morbilidad. De acuerdo con este aspecto, es recomendado por varias guías nacionales e internacionales, y su implementación es potencialmente beneficiosa tanto en la prevención como en el control de la hipertensión arterial.

Palabras clave: Hipertensión, Enfoques alimenticios para detener la hipertensión, Salud pública, Dieta mediterrânea.

\section{INTRODUÇÃO}

Ao realizar uma análise do perfil demográfico e de morbimortalidade em todas as regiões do mundo, foram observadas significativas evidências, ainda que com diferentes magnitudes, da mudança do padrão de adoecimento da população no século XXI. Foi registrada uma redução da taxa de fecundidade e de mortalidade por doenças infecciosas e um aumento da expectativa de vida e das mortes por doenças crônicas, principalmente as doenças cardiovasculares. No Brasil, a população idosa passou de 4,7\%, em 1960, para 10,8\%, em 2010, o que, em números absolutos, o que representa um acréscimo, nesse período, de 3,3 milhões de idosos para cerca de 20,5 milhões de pessoas (GONÇALVES K, et al., 2017).

Nesse contexto, também é notável o aumento da progressão da Hipertensão Arterial como fator de base para o desenvolvimento dessas comorbidades. Sendo assim, diversos estudos desenvolvidos identificaram uma série de fatores de risco que contribuem para o desfecho da maior ocorrência de eventos cardiovasculares na população em geral e a hipertensão arterial é um dos mais comuns entre eles. No mundo, a hipertensão é uma das principais causas de morte prematura. Estima-se que um terço da população adulta do mundo possua hipertensão, com uma prevalência estimada em aproximadamente um bilhão, o que é projetado para aumentar. O aumento previsto da hipertensão globalmente é atribuído ao aumento da idade, bem como à disponibilidade e ingestão de uma dieta ocidentalizada associada à adoção de um estilo de vida sedentário, levando à obesidade. O maior risco de hipertensão associado a fatores alimentares e de estilo de vida não saudáveis destaca a importância de intervir em fatores ambientais durante o início e a progressão da hipertensão (SHIMBO D, 2016).

Não Obstante, se torna necessário enfatizar a necessidade da adesão ao tratamento, visto que as medidas farmacológicas impactam positivamente nos domínios mental, físico e escore total da qualidade de vida de paciente hipertensos. O principal objetivo do tratamento da hipertensão arterial é a redução da morbidade e da mortalidade cardiovasculares, sendo assim, os anti-hipertensivos devem não só reduzir a pressão arterial, mas também os eventos cardiovasculares fatais e não fatais. Contudo é importante considerar os malefícios que a polifarmacia pode trazer, preferindo sempre que possível inserir uma medida conservadora associada ao tratamento medicamentoso.

A associação desregulada de múltiplos medicamentos cria regimes terapêuticos complexos, o que facilita a ocorrência de erros de medicação, interações medicamentosas e reações adversas, estas, em geral, decorrentes de ação central, podem se manifestar como sonolência, sedação, boca seca, fadiga, hipotensão postural e disfunção sexual. Em meio a isso é importante considerar a existência de uma medida complementar, a fim de intensificar a ação medicamentosa no organismo. 
O incentivo a mudanças do habito de vida do paciente pode ser uma tarefa difícil para o profissional da saúde, mas dentro da atenção primaria torna- se mais fácil estabelecer um vínculo de confiança, intensificando a relação médico- paciente e facilitando a adesão ao tratamento (CAETANO AC, et al., 2016; RIBEIRO A, et al., 2010; RODRIGUES MC, et al., 2016).

Dentro das mudanças no estilo de vida se encaixa uma proposta alimentar relativamente recente, a Dietary Approaches to Stop Hypertension (DASH) é uma técnica dietética desenvolvida na década de 1990, após pesquisadores norte-americanos chegarem à conclusão de que, fatores dietéticos, como o alto índice de sódio, ingestão de bebidas alcoólicas e o excesso de peso estavam diretamente relacionados com o desenvolvimento da hipertensão.

O primeiro ensaio clínico acerca da dieta DASH foi publicado em 1997, este dividiu uma amostra de adultos americanos, hipertensos ou não, em três grupos: o primeiro, grupo controle, foi orientado a seguir o padrão de dieta americano, o segundo a uma dieta rica em frutas e verduras (FV) e o terceiro seguiu a dieta DASH. O resultado mostrou uma redução significativa da pressão arterial sistólica e diastólica nos participantes que seguiram a dieta DASH comparados ao grupo controle, inclusive maior do que os participantes que seguiram a dieta FV, principalmente se considerarmos os participantes hipertensos leves.

A partir desse primeiro resultado positivo, novos estudos e ensaios começaram a ser realizados para avaliar melhor a eficácia da dieta no controle da hipertensão arterial.Com respeito a seus componentes, a DASH é um padrão alimentar baseado em frutas, vegetais, laticínios sem gordura ou com pouca gordura, grãos integrais, nozes e legumes. Além disso, também contém alimentos ricos em potássio, magnésio e fibras e limita a gordura total e saturada, colesterol, carnes vermelhas e processadas, doces, açúcares adicionados e bebidas açucaradas. (BRICARELLO L, et al., 2020; CHIU S, et al., 2016; DE PERGOLA G, et al., 2018).

Tendo em vista essa grande oferta de alimentos ricos em fibras, polifenóis, além de micro e macronutrientes essenciais, a aderência correta e assídua à dieta DASH pode proporcionar níveis pressóricos menores, além de ajudar na prevenção de doenças secundárias a essa condição. Assim, a implementação dessa dieta, como forma de manejo de pacientes hipertensos na atenção primária do Brasil parece ser uma estratégia interessante e com bons resultados, como já é sugerido pelo governo de Hong Kong, desde 2010 (WANG HH, et al., 2015; GAO M, et al., 2018).

De acordo com a Organização Mundial da Saúde (OMS), a hipertensão é o principal fator de risco para doenças cardiovasculares (DCV) e mortalidade de todas as causas, representando atualmente cerca de $30 \%$ de todas as mortes globais. Por esse motivo a dieta DASH tem sido recomendada nas diretrizes internacionais para hipertensão. Paralelamente, de acordo com as estimativas da OMS, aproximadamente $80 \%$ das mortes por DCV poderiam ser evitadas através do controle de certos fatores do estilo de vida relacionados a um conjunto de fatores de risco intermediários de DVC. A dieta está incluída entre os fatores do estilo de vida com maior impacto nos fatores de risco para DCV e no risco de desenvolvimento de DCV. Além disso, ela pode ser um forte aliado no combate da síndrome metabólica, podendo oferecer desfechos favoráveis para jovens, e também adultos que apresentam essa condição (ASGHARI G, et al., 2016; De PERGOLA G, et al., 2018; TSIOUFIS C, et al., 2018; WONG MC, et al., 2018).

No Brasil, a hipertensão é umas uma das principais causas de morbimortalidade. No país, a prevalência de hipertensão atinge $23,3 \%$ da população adulta. Segundo a VII Diretriz Brasileira de Hipertensão a adesão à dieta DASH reduz a pressão arterial sistólica e diastólica em aproximadamente $6,7 / 3,5 \mathrm{mmHg}$, por isso é hoje recomendada como parte do tratamento não medicamentosos da hipertensão. Em suma, ela possui importante papel de melhorar a função endotelial e oferecer um benefício considerável contra o risco de hipertensão e DCV (Doença Cardiovascular) (DE PERGOLA G, et al, 2018; JENKINS DJ, et al., 2015; LIMA ST, et al., 2013; MALACHIAS MVB, et al., 2016).

Diante do contexto, o presente artigo busca, através de uma revisão narrativa da literatura científica, comprovar a importância da dieta DASH no manejo da hipertensão arterial e por meio das informações apresentadas correlacionar a dieta DASH a um tratamento benéfico e plausível para pacientes hipertensos na atenção primária, visando a redução dos níveis pressóricos e aproveitando de sua função cardioprotetora para a prevenção de doenças associadas a esse grupo. 


\section{REVISÃO BIBLIOGRÁFICA}

A fim de elaborar uma tabela com os principais resultados obtidos pela literatura, foi realizada uma busca na base de dados Medical Literature Analysis and Retrieval System Online (MEDLINE), por meio dos seguintes descritores: "Dietary Approaches To Stop Hypertension" e "Primary Health Care". Após uma seleção inicial, foi feita uma leitura prévia do resumo de cada um destes artigos, sendo descartados aqueles que não atendiam de imediato os objetivos dessa revisão, ou seja, verificar a eficácia da dieta DASH no tratamento de pacientes hipertensos na atenção primária. Dessa forma, foram selecionados 7 ensaios clínicos randomizados (Tabela 1).

Tabela 1 - Achados bibliográficos.

\begin{tabular}{|c|c|c|}
\hline Autores (ano) & $\begin{array}{l}\text { Principais } \\
\text { características da } \\
\text { amostra }\end{array}$ & Principais resultados \\
\hline $\begin{array}{l}\text { Lima } S \text {, et al. } \\
(2013)\end{array}$ & $\begin{array}{l}\text { ldade superior a } 20 \\
\text { anos, sem doença de } \\
\text { base. }\end{array}$ & $\begin{array}{l}\text { A dieta } \mathrm{DASH}^{*} \text { com menor teor de sódio }(1500 \mathrm{mg}) \text { exibiu } \\
\text { uma redução de } 11,9 \mathrm{mmHg} \text { na PAS }{ }^{* \star * \star *} \text { quando } \\
\text { comparada com a dieta americana tradicional com maior } \\
\text { teor }(3300 \mathrm{mg}) \text {. }\end{array}$ \\
\hline $\begin{array}{l}\text { Chiu S, et al. } \\
(2016)\end{array}$ & $\begin{array}{l}\text { ldade inferior a } 21 \\
\text { anos, com } \mathrm{PAD}^{* * * *} \\
\text { média entre } 80 \text { e } 95 \\
\text { mm } \quad \mathrm{Hg} \text { e } \\
\mathrm{PAS}^{* * * * *}<160 \mathrm{~mm} \mathrm{Hg} \text {. }\end{array}$ & $\begin{array}{l}\text { A dieta DASH }{ }^{*} \text { foi fator de redução da pressão arterial. } \\
\text { Contudo, sua variante HF-DASH }{ }^{* *} \text {, com menor restrição } \\
\text { dietética e de gordura reduziu também as concentrações } \\
\text { de triglicérides plasmáticos e VLDL }{ }^{* * * * * *} \text { sem aumentar } \\
\text { significativamente o colesterol } \mathrm{LDL}^{* * *} \text {. }\end{array}$ \\
\hline $\begin{array}{l}\text { Augustin B, et al. } \\
\text { (2015) }\end{array}$ & Dislipidemia. & $\begin{array}{l}\text { Não foi observada redução da pressão arterial do grupo } \\
\text { em que foi introduzida a DASH*, provavelmente porque a } \\
\text { dieta terapêutica básica dos participantes já era } \\
\text { semelhante. Apesar disso, verificou-se que o aumento da } \\
\text { conformidade com os componentes da dieta } D_{S A}{ }^{*} \text { no } \\
\text { grupo controle ainda estava associado à redução da } \\
\text { pressão arterial. }\end{array}$ \\
\hline $\begin{array}{l}\text { Asghari G, et al. } \\
(2016)\end{array}$ & $\begin{array}{lr}\text { Crianças } & e \\
\text { adolescentes } & (6-18 \\
\text { anos). } & \end{array}$ & $\begin{array}{l}\text { A incidência de hipertensão, glicemia plasmática em jejum } \\
\text { e gordura abdominal diminuíram com a maior adesão da } \\
\text { dieta } \mathrm{DASH}^{*} \text {. }\end{array}$ \\
\hline $\begin{array}{l}\text { Soltani S, et al. } \\
\text { (2016) }\end{array}$ & $\begin{array}{l}\text { Sobrepeso } \\
\text { obesidade. }\end{array}$ & $\begin{array}{l}\text { A dieta DASH* pode ser uma escolha melhor do que dietas } \\
\text { típicas de baixa caloria na redução de peso e de gordura } \\
\text { corporal, particularmente para indivíduos com sobrepeso e } \\
\text { obesidade. }\end{array}$ \\
\hline $\begin{array}{l}\text { Farhadneja } \mathrm{H} \text {, et } \\
\text { al. (2019) }\end{array}$ & $\begin{array}{l}\text { Idade superior a } 20 \\
\text { anos. }\end{array}$ & $\begin{array}{l}\text { Indivíduos com sobrepeso e obesidade apresentaram } \\
\text { melhora do perfil metabólico ao aderir o padrão alimentar } \\
\text { DASH }^{*} \text {. }\end{array}$ \\
\hline $\begin{array}{l}\text { Wong MCS, et al. } \\
(2016)\end{array}$ & $\begin{array}{l}\text { Hipertensão grau } 1 \text { e } \\
\text { idade entre } 40 \text { e } 70 \\
\text { anos. }\end{array}$ & $\begin{array}{l}\text { Tanto no grupo em uso da dieta } \mathrm{DASH}^{*} \text { quanto no grupo } \\
\text { controle houve melhora significativa do risco } \\
\text { cardiovascular em } 10 \text { anos. Contudo, ambos os grupos } \\
\text { não apresentaram diferença importante no risco } \\
\text { cardiovascular em } 6 \text { meses. }\end{array}$ \\
\hline
\end{tabular}

Legenda: DASH (Dietary Approaches to Stop Hypertension) ${ }^{*}$, HF-DASH (Higher fat - Dietary Approaches to stop hypertension), LDL (Low Density Lipoprotein) ${ }^{\star * *}$, PAD (Pressão Arterial Diastólica) ${ }^{* \star * *}$, PAS (Pressão Arterial Sistólica) ${ }^{* * * *}$, VLDL (Very Low Density Lipoprotein) $)^{* * * * * *}$.

Fonte: Rodrigues LL, et al., 2020.

A dieta DASH é uma das modificações dietéticas mais amplamente prescritas em diversos países para reduzir a pressão arterial e o risco de doença cardiovascular. Comparada com a dieta ocidental típica, apresenta menor consumo de colesterol LDL, devido em parte à limitação de ácidos graxos saturados. Portanto acredita-se que seu menor teor de gordura saturada possa contribuir para reduzir o risco de doença cardiovascular (CHIU S, et al., 2016). 
Considerando-se que a hipertensão arterial sistêmica é hoje uma das comorbidades mais prevalentes na saúde pública, atingindo mais de 36 milhões de adultos e cerca de $60 \%$ dos idosos no Brasil, é cada vez mais necessário que os profissionais da saúde busquem estratégias que ajudem a controlar ou até mesmo evitar essa condição já na atenção primária (MALACHIAS MVB, et al., 2016). Existem evidências de que fatores de risco como IMC de alto risco (> 27,5 kg / $\mathrm{m}^{2}$ ), tabagismo, bebidas alcoólicas, regime alimentar não saudável, refeições irregulares diárias e presença de histórico parental de hipertensão foram independentemente e significativamente associadas à hipertensão grau 1.

Indivíduos do sexo masculino e do sexo feminino com quatro ou mais dos fatores de risco supracitados, tiveram respectivamente sete e treze vezes a probabilidade prevista de ter hipertensão grau 1 quando comparados a indivíduos sem fatores de risco. Os fatores de ingestão alimentar foram associados à hipertensão grau 1 de forma significativa, ressaltando a importância sobre a incorporação de intervenções relacionadas à dieta com base na abordagem DASH para o tratamento da hipertensão nos serviços de atenção primária (WANG HH, et al., 2015).

Dessa forma, vários estudos foram performados para verificar a aplicabilidade da implementação dessa dieta como uma alternativa viável para o manejo clínico da hipertensão. Alguns estudos realizados na atenção primária com hipertensos se mostraram positivos, apresentando como resultado uma maior redução da pressão arterial e menor excreção de sódio na urina entre os participantes que seguiram a dieta DASH se comparados ao controle. (LIMA ST, et al., 2013). O estudo DASH original, realizado com 133 pacientes hipertensos, comparou os efeitos de uma dieta tradicional americana com uma rica em frutas e vegetais. Notavelmente, ambas as dietas continham $3000 \mathrm{mg} / \mathrm{dia}$ de sódio $(\mathrm{Na})$.

No seguimento de 8 semanas, foram observadas uma redução de $11,4 \mathrm{mmHg}$ na pressão arterial sistólica e uma redução de $5,5 \mathrm{mmHg}$ na pressão arterial diastólica entre os usuários da Dieta DASH. Em um ensaio clínico subsequente, os efeitos de uma dieta americana tradicional e de uma dieta DASH nos níveis de pressão arterial foram comparados. A dieta DASH com menor teor de $\mathrm{Na}(1500 \mathrm{mg})$ exibiu uma redução de $11,9 \mathrm{mmHg}$ na PAS quando comparada com a dieta americana tradicional com maior teor de $\mathrm{Na}$ (3300mg) (WANG HH, et al., 2015; LIMA ST, et al., 2013). Além disso, a dieta DASH se mostrou uma boa opção para controle de peso, particularmente em indivíduos com sobrepeso e obesidade. Adultos, submetidos a um estudo perderam mais peso em 8 a 24 semanas e reduziram mais o Índice de Massa Corpórea (IMC) em 8 a 52 semanas e a circunferência abdominal em 24 semanas em comparação com o grupo controle. O DASH baixo em calorias mostrou uma redução ainda maior do peso quando comparado com outras dietas de baixa energia. Ainda, o efeito foi maior em participantes com sobrepeso / obesidade quando comparados com dietas típicas (ocidentais ou usuais da população).

Outra questão verificada por esse estudo, foi que esse padrão alimentar, além de reduzir a pressão arterial, contribuiu para melhorar o índice glicêmico, reduzir o risco de DCV e de câncer. Cada quilograma na redução do peso pode resultar em uma diminuição de $1 \mathrm{mmHg}$ na pressão arterial sistólica e diastólica (SOLTANI S, et al., 2016). Além de tudo, outro estudo evidenciou que a maior adesão à dieta estilo DASH desempenha um papel favorável na melhora metabólica de indivíduos com peso adequado, achados observados entre participantes mais jovens, e não idosos. Ainda, foi observada uma associação significativa entre dieta DASH e a melhora do perfil metabólico em obesos com idade $\geq 20$ anos. Resultados demonstraram que a dieta DASH está inversamente relacionada a danos metabólicos em pessoas com sobrepeso e obesidade (FARHADNEJAD H, et al., 2019).

A fim de facilitar essa adesão, foram desenvolvidas outras estratégias, como a "Higherfat -Dietary Approaches to stop hypertension" (HF-DASH), uma modificação com mais gordura e menos carboidrato. Essa mostrou reduzir a PA sistólica e diastólica em uma extensão semelhante à dieta DASH, indicando que os componentes da dieta responsáveis pela redução da PA foram retidos na dieta HF-DASH.

Houve também redução das concentrações plasmáticas de triglicérides e VLDL (Very Low Density Lipoprotein), sem aumentar significativamente o colesterol LDL (Low Density Lipoprotein). Com isso, presume-se que esse padrão alimentar, que permite uma ingestão mais liberal de gordura total e saturada em conjunto com uma limitação moderada da ingestão de carboidratos, principalmente de sucos e açúcares 
de frutas, resulta em melhora significativa do perfil metabólico, além de melhor adesão por se tratar de uma dieta menos restrita que a DASH (CHIU S, et al., 2016).

Ademais, quando se fala em atenção primária, é necessário, também, pensar em estratégias para crianças e adolescentes. O resultado de um estudo foi capaz de associar uma maior adesão a dieta DASH com menores índices de pressão arterial sistólica e de gordura abdominal entre jovens, sendo assim, uma possível estratégia para frear o desenvolvimento de hipertensão nessa faixa etária. Apesar do mecanismo exato de como os nutrientes da dieta podem levar a esses resultados ainda não serem totalmente conhecido, especulase que a grande quantidade de antioxidantes, fibras, maior consumo de potássio e menor consumo de sódio possa estar relacionados a esse desfecho.

Dessa forma, verificou-se que a incidência de hipertensão, glicemia plasmática em jejum e gordura abdominal diminuíram com maior adesão à dieta DASH. (ASGHARI G, et al., 2016). Paralelamente, outras pesquisas realizadas também confirmam sua eficácia. Tanto no grupo controle quanto no grupo em uso da DASH houve melhora significativa do risco cardiovascular em 10 anos. Os dois grupos, no entanto, não evidenciaram diferença importante no risco cardiovascular em 6 meses. As melhorias no perfil lipídico e IMC foram observadas entre todos os indivíduos, mas não foram detectadas diferenças significativas entre os grupos intervenção e controle (WONG MCS, et al., 2016).

Entretanto, outros estudos evidenciam resultados diferentes. Apesar de, por um lado, a dieta DASH ser relacionada a redução de níveis pressóricos e de peso corporal a curto prazo, poucos estudos demonstram seus efeitos a longo prazo. Sabendo disso, uma pesquisa na atenção primária em Hong Kong acompanhou pacientes hipertensos por um ano e, apesar da redução dos níveis pressóricos entre os pacientes que seguiram a dieta DASH, não houveram benefícios adicionais se comparados ao grupo controle, que apenas seguiram orientações usuais dos médicos, questionando-se, assim, a real aplicabilidade da dieta (WONG MCS, et al., 2016). Em concordância com esse ponto de vista, em um estudo realizado por Augustin B, et al. (2015); durante 24 semanas, uma dieta do tipo DASH foi comparada a um portifólio dietético, baseado em componentes alimentares saudáveis, como nozes, proteínas de soja, óleos vegetais e fibra viscosa, não sendo observadas evidências de benefício do controle pressórico nos pacientes submetidos à DASH. O portfólio evidenciou uma maior redução das pressões arteriais sistólica, diastólica e arterial média em comparação ao controle em 2,1 mm Hg (IC95\%, 4,2 a -0,1 mm Hg) $(p=0,056), 1,8 \mathrm{~mm} \mathrm{Hg}(\mathrm{IC}, 3,2$ a 0,4 mm $\mathrm{Hg})(p=0,013)$ e $1,9 \mathrm{~mm} \mathrm{Hg}(\mathrm{IC}, 3,4$ a $0,4 \mathrm{~mm} \mathrm{Hg})(p=0,015)$, respectivamente. Essa redução da pressão arterial, combinada com o efeito de melhora do perfil lipídico do portfólio alimentar, evidencia uma redução significativa no escore de risco de doença cardiovascular.

Contudo, esse estudo apresenta um importante viés, mesmo tendo adesão de mais de $100 \%$, a dieta do tipo DASH falhou em reduzir a pressão sanguínea provavelmente porque a dieta terapêutica de base dos participantes já possuía muitos dos recursos do grupo estudo (conformidade acima de $60 \%$ ), além de a pressão arterial dos participantes já estar em níveis adequados de aproximadamente $119 / 73 \mathrm{~mm} \mathrm{Hg}$, portanto, subestimando seu verdadeiro efeito.

Também é importante ressaltar que o tratamento de pacientes hipertensos deve-se basear não apenas na mudança de dieta, mas também em uma prática regular de atividades físicas, como sugere um estudo realizado em uma população norte americana, que mostrou que a dieta DASH foi capaz de promover um maior índice de participantes metabolicamente saudáveis apenas em menores de 45 anos, enquanto pacientes mais idosos não obtiveram os mesmos resultados. Por outro lado, entre aqueles maiores de 45 anos que conseguiram manter um nível de atividade física recomendado os resultados foram semelhantes aos mais jovens (PARK YM, et al., 2016). Apesar de parecer uma estratégia eficiente, é preciso verificar melhor a aplicabilidade da dieta DASH a longo prazo, levando em conta a sua eficácia sobre outras medidas de tratamentos não medicamentosos da hipertensão já consagrados, buscando entender melhor, assim, 0 real custo-benefício de sua prática.

Apesar das evidências trazidas pelos estudos, existe um importante viés a ser considerado ao abordar a implementação dessa dieta na atenção primária, que é a significativa disparidade econômica presente na população brasileira. Logo, é necessário considerar as limitações da sua implementação integral, já que os 
seus componentes tendem a apresentar um valor de aquisição consideravelmente maior do que os itens de uma dieta convencional, sendo, assim, necessário avaliar individualmente ,de acordo com o perfil socioeconômico de cada paciente, a real possibilidade e custo benefício da aplicação desta técnica dietética.

\section{CONSIDERAÇÕES FINAIS}

O uso da dieta DASH consiste em uma importante medida para controle dos níveis pressóricos, podendo ser implementada na atenção primária de saúde, já que a terapia medicamentosa individualizada tem uma eficácia menor comparada à associação da DASH como adjuvante no tratamento anti-hipertensivo. Sendo assim, constitui uma medida conservadora que propicia a melhora da qualidade de vida no paciente hipertenso, evitando em muitos casos a evolução de seu quadro. Paralelamente, outro benefício observado foi a redução da incidência de doença cardiovascular, em decorrência do controle pressórico nos pacientes que aderiram a essa terapêutica, sendo assim, dieta DASH se mostra uma boa alternativa a ser implementada no plano terapêutico do paciente com sobrepeso ou obesidade em detrimento de dietas típicas de baixa caloria, a fim de reduzir o peso e a gordura corporal. Em contrapartida, não se pode negar os desafios ao incentivo da implementação dessa dieta aos pacientes da atenção primária brasileira devido às condições socioeconômicas. Por isso, deve ser avaliado individualmente a prescrição da dieta, em alguns casos podendo ser flexibilizada.

\section{REFERÊNCIAS}

1. ASGHARI G, et al. Dietary Approaches to Stop Hypertension (DASH) Dietary Pattern Is Associated with Reduced Incidence of Metabolic Syndrome in Children and Adolescents. The Journal Of Pediatrics, 2016; 174: 178-184.

2. BAKONINA Y. Global Status Report on Non-Communicable Diseases. World Health Organization, 2014; 1:1-9.

3. BRICARELLO L, et al. Abordagem dietética para controle da hipertensão: reflexões sobre adesão e possíveis impactos para a saúde coletiva.Ciência e saúde coletiva, 2020; 25(4): 1421-1432.

4. CAETANO AC, et al.Quality of life and treatment adherence in hypertensive patients: systematic review with metaanalysis. Revista de Saúde Pública, 2016; 50:71.

5. CHIU S, et al. Comparison of the DASH (Dietary Approaches to Stop Hypertension) diet and a higher-fat DASH diet on blood pressure and lipids and lipoproteins: a randomized controlled trial. Am J Clin Nutr. 2016;103(2):341-347.

6. DE PERGOLA G, et al. Influence of Mediterranean Diet on Blood Pressure. Nutrients. 2018;10(11):1700.

7. FARHADNEJAD H, et al. The association of Dietary Approach to Stop Hypertension (DASH) diet with metabolic healthy and metabolic unhealthy obesity phenotypes. Scientific Reports, 2019; 9:1-7.

8. GAO M, et al. Trajetórias da adesão à dieta mediterrânea e risco de hipertensão na China: resultados do estudo CHNS, 1997-2011. Nutrientes, 2018; 10 (12): 2014.

9. GONÇALVES K, et al. Polifarmácia em idosos: um estudo de base populacional. Revista Brasileira de Epidemiologia, 2017; 20(2):335-344.

10. JENKINS DJ, et al. The effect of a dietary portfolio compared to a DASH-type diet on blood pressure. Nutrition, Metabolism and Cardiovascular Diseases, 2015; 25 (12): 1132-9.

11. LIMA ST, et al. Dietary approach to hypertension based on low glycaemic index and principles of DASH (Dietary Approaches to Stop Hypertension): a randomised trial in a primary care service. British Journal of Nutrition, $2013 ; 110: 1472-1479$.

12. MALACHIAS MVB, et al. 7ª Diretriz Brasileira de Hipertensão Arterial.Arquivos Brasileiros de Cardiologia, 2016; 107(3):1104.

13. MOK RY, et al. Factors associated with grade 1 hypertension: implications for hypertension care based on the Dietary Approaches to Stop Hypertension (DASH) in primary care settings. BMC Fam Pract. 2015;16:26.

14. PARK YMM, et al. Mediterranean diet, Dietary Approaches to Stop Hypertension (DASH) style diet, and metabolic health in US adults. Clinical nutrition, 2017; 36(5): 1301-1309.

15. RIBEIRO A, et al.Tratamento medicamentoso.Brazilian Journal of Nephrology, 2010; 32:S1.

16. RODRIGUES MC, et al.Drug-drug interactions and adverse drug reactions in polypharmacy among older adults: an integrative review. Rev Lat Am Enfermagem, 2016; 24: e2800.

17. SHIMBO D. Dietary and lifestyle factors in hypertension. Journal of Human Hypertension, 2016; 30:571-572

18. SOLTANI S, et al. The effect of dietary approaches to stop hypertension (DASH) diet on weight and body composition in adults: a systematic review and meta-analysis of randomized controlled clinical trials. Obesity reviews, 2016; 17(5): 442-454.

19. TSIOUFIS C, et al. The Mediterranean and the DASH dietary patterns: Insights into their role in cardiovascular disease prevention. Hellenic J Cardiol, 2018; 59 (2): 134-135.

20. WANG HH, et al. Factors associated with grade 1 hypertension: implications for hypertension care based on the Dietary Approaches to Stop Hypertension (DASH) in primary care settings. BMC Family Practice, 2015; 16: 26.

21. WONG MC, et al. Dietary counselling has no effect on cardiovascular risk factors among Chinese Grade 1 hypertensive patients: a randomized controlled trial. European Heart Journal, 2015; 36(38): 2598-2607.

22. WONG MC, et al. The effectiveness of Dietary Approaches to Stop Hypertension (DASH) counselling on estimated 10-year cardiovascular risk among patients with newly diagnosed grade 1 hypertension: A randomised clinical trial. International journal of cardiology, 2016; 224: 79-87. 